\title{
Distal Pancreatectomy En Bloc With Splenectomy, Celiac Axis and Portal Vein Resection Followed by Arterial Reconstruction Using a Cadaveric Graft - A Case Report and Literature Review
}

\author{
VLADISLAV BRASOVEANU ${ }^{1,2}$, ION BARBU $^{1}$, FLORIN ICHIM $^{1}$, \\ IRINA BALESCU ${ }^{3}$ and NICOLAE BACALBASA ${ }^{4,5,6}$ \\ 1 "Dan Setlacec" Center of Gastrointestinal Diseases and Liver Transplantation, \\ Fundeni Clinical Institute, Bucharest, Romania; \\ 2 "Titu Maiorescu” University of Medicine and Pharmacy, Bucharest, Romania; \\ 3 "Ponderas" Academic Hospital, Bucharest, Romania; \\ 4 "Carol Davila" University of Medicine and Pharmacy, Bucharest, Romania; \\ ${ }^{5}$ Center of Excellence in Translational Medicine, Fundeni Clinical Institute, Bucharest, Romania; \\ 6“I. Cantacuzino” Clinical Hospital, Bucharest, Romania
}

\begin{abstract}
Background/Aim: Locally advanced pancreatic body tumors invading the celiac axis and the portal vein have been considered since long as unresectable lesions; however, due to improvement of surgical techniques, in certain cases surgery with curative intent might be taken in consideration. Case Report: We present the case of a 48year-old female investigated for epigastric pain that was diagnosed with a locally invasive pancreatic body tumor. The patient was submitted to computed tomography which revealed the presence of a locally advanced pancreatic tumor with no demarcation line with the celiac axis and the portal vein. The endoscopic ultrasound raised the suspicion of malignancy and retrieved a biopsy which demonstrated the presence of a pancreatic adenocarcinoma. The patient was submitted to surgery, distal pancreatectomy en bloc with splenectomy, celiac axis and portal vein resection was performed; the hepatic artery was reconstructed by placing a cadaveric graft while the portal vein was sutured per primam by an end to end anastomosis. The postoperative outcome was favorable, and the patient was discharged in the $10^{\text {th }}$ postoperative day. Conclusion: Multiple vascular
\end{abstract}

This article is freely accessible online.

Correspondence to: Irina Balescu, "Ponderas" Academic Hospital, Nicolae Caramfil 85a Street, Bucharest, Romania. Tel: +40 724077709, e-mail: irina.balescu@ ponderas-ah.ro

Key Words: Pancreatic body adenocarcinoma, celiac axis resection, portal vein resection, cadaveric graft. resections followed by reconstructions might be needed in order to achieve resection with negative margins in patients with pancreatic body tumors.

Pancreatic cancer remains the most aggressive malignancy, affecting patients worldwide, whilst being responsible for the lowest rates of 5-year survival (1). Moreover, among pancreatic malignancies more than $75 \%$ of cases presenting pancreatic body and tail lesions are considered unresectable at the time of diagnostic due to the presence of local extension at the level of the greater vessels or due to the presence of metastatic disease $(2,3)$. While the presence of venous invasion no longer represents a criterion of unresectable disease, similar rates of survival have been reported after pancreatic resection as a stand-alone procedure and pancreatic resections en bloc with portal vein resection followed by reconstruction. However, the presence of arterial invasion represents a hot subject of debate (4). Although initially the presence of celiac axis or superior mesenteric artery is considered a sign of unresectable disease, distal pancreatectomy en bloc with celiac axis resection and hepatic artery reconstruction have been proven during the last decades to be an effective procedure in order to achieve negative resection margins and offer a chance for long-term survival $(5,6)$.

\section{Case Report}

A 48-year-old female with no significant medical history was investigated for weight loss of $12 \mathrm{~kg}$ in the last three months and epigastric pain irradiating to the dorsal area. In the 
meantime, no other signs of symptoms such as nausea, vomiting or alteration of the digestive habits were reported.

The patient was initially submitted to an abdominal ultrasound which raised the suspicion of a pancreatic body tumor, so the imagistic investigations were further completed by a computed tomography and magnetic resonance imaging which confirmed the presence of a $4 / 5 / 4 \mathrm{~cm}$ pancreatic body tumor invading the celiac axis and the portal vein; in the meantime the absence of distant metastases was also demonstrated. The patient was further submitted to an endoscopic ultrasound which retrieved a pancreatic biopsy which demonstrated the presence of a well differentiated pancreatic adenocarcinoma. Laboratory studies demonstrated the presence of an increased level of the antigen carbohydrate $19-9$ - measuring $450 \mathrm{ng} / \mathrm{ml}$ with normal liver function. In this context, due to the good biological status and the absence of distant metastases the patient was submitted to surgery; therefore the tumor was resected en bloc with distal pancreatectomy, splenectomy, celiac axis resection and portal vein resection. The continuity of the portal vein was reestablished through a side-to-side anastomosis due to the relatively low length of the resected vein; in the meantime, once the celiac axis was clamped, a very weak blood flow was encountered at the level of the common hepatic artery. Therefore, the common hepatic artery was reconstructed by using a cadaveric venous graft from the iliac vein which was anastomosed between the common hepatic artery stump and the abdominal aorta (Figures 1 and 2). The length of surgery was of 320 minutes with an estimated blood loss of $800 \mathrm{ml}$. The postoperative outcome was uneventful; the Doppler ultrasound revealed arterial reconstruction while the biological tests performed in the fifth postoperative day revealed normalization of the liver tests; the patient was discharged in the 10th postoperative day. The histopathological studies confirmed the presence of a well differentiated pancreatic adenocarcinoma invading the celiac axis and the portal vein; in the meantime, the negativity of the resection margins was also demonstrated providing a radical character of the surgical procedure.

\section{Discussion}

Due to the fact that pancreatic body tumors remain asymptomatic for a long period of time a significant number of cases will be diagnosed in advanced stages of the disease; therefore, these patients will be diagnosed after a long period of local evolution of the tumoral process that will lead to the apparition of vascular invasion of the surrounding structures $(7,8)$. Although initially it has been considered that the presence of vascular invasion should be considered as a criterion of unresectable lesion, improvement of the surgical techniques led to a higher rate of association of vascular resections; in the meantime the benefit of survival also became evident (9-15). Therefore, patients submitted to standard pancreatic resections reported similar long-term outcomes when compared to cases submitted to pancreatic resections en bloc with portal vein resection. Moreover, in certain cases a benefit in terms of survival was also observed in selected cases submitted to concomitant arterial resection; therefore, the presence of arterial and venous invasion was no longer considered a formal contraindication for surgery (16-19).

When it comes to the association of celiac axis invasion, cases diagnosed with locally invasive pancreatic body adenocarcinoma invading the celiac axis seem to benefit from en bloc removal of the tumor with distal pancreatectomy, splenectomy, celiac axis and nerve plexus around the major vessels (6). The procedure is inspired by the surgical approach initially proposed by Appleby in order to treat gastric cancer invading the celiac axis; at that moment the Appleby procedure consisted of total gastrectomy en bloc with distal pancreatectomy and celiac axis resection (20). Further on the procedure was modified and proposed in order to treat locally invasive pancreatic body tumors with celiac axis invasion; in this context the tumor is resected en bloc with distal pancreatectomy and celiac axis resection. Although anatomical studies came to demonstrate that the presence of collateral circulation between the hepatobiliary system and the superior mesenteric artery through an intact pancreaticoduodenal arcade might reduce the risk of perioperative complications, the risks of gastric and hepatic ischemia is still significant (21).

In order to identify which cases might benefit most from this aggressive surgical approach, certain authors created different prognostic preoperative scoring systems; therefore in the study conducted by Miura et al., 50 patients submitted to distal pancreatectomy en bloc with celiac axis resection were included (22). According to that study, patients with intraoperative blood loss higher than $940 \mathrm{ml}$, with lower than $150 \times 109 / 1$ levels of preoperative platelet count, higher than $0.4 \mathrm{mg} / \mathrm{dl}$ levels of $\mathrm{C}$ reactive protein and higher than 300 $\mathrm{U} / \mathrm{ml}$ preoperative levels of antigen carbohydrate 19-9 were more likely to develop preoperative complications. In this respect the Japanese authors accorded a score of one point for each above mentioned parameter and demonstrated that the median overall survival as well as the one- and five-year overall survival were significantly influenced by the preoperative score; therefore, the median overall survival was of 50.6 months for cases with preoperative score of 0 , 22.3 months for those with preoperative score of 1 and 7.7 months for those with higher scores. Therefore, the authors concluded that this score could be a useful tool to select cases that will benefit most from this demanding procedure. In the meantime, the authors reported the fact that 32 out of the 50 cases also associated portal vein resection; however, this factor failed to have a significant impact on the long- 


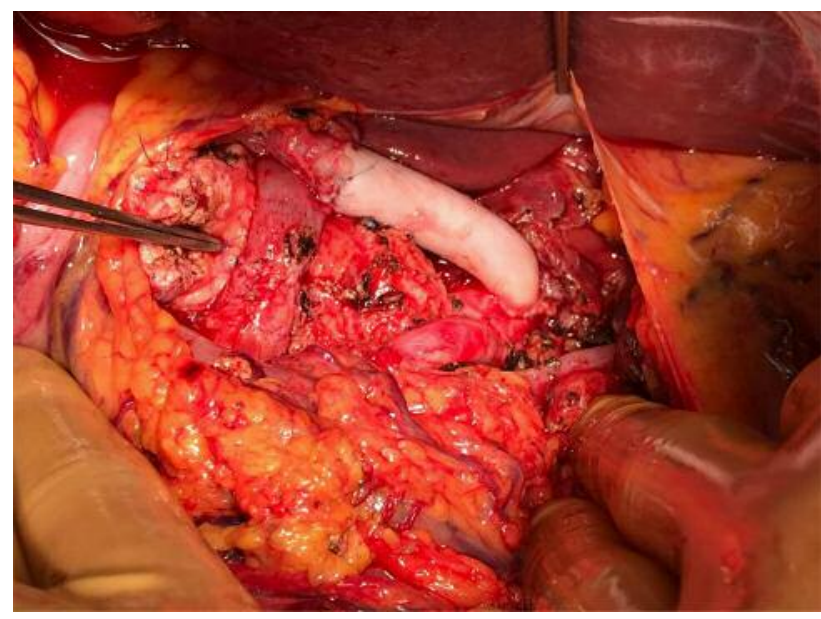

Figure 1. The final aspect after pancreatic resection en bloc with celiac axis and portal vein resection - the grasper is pointing out the portal suture.

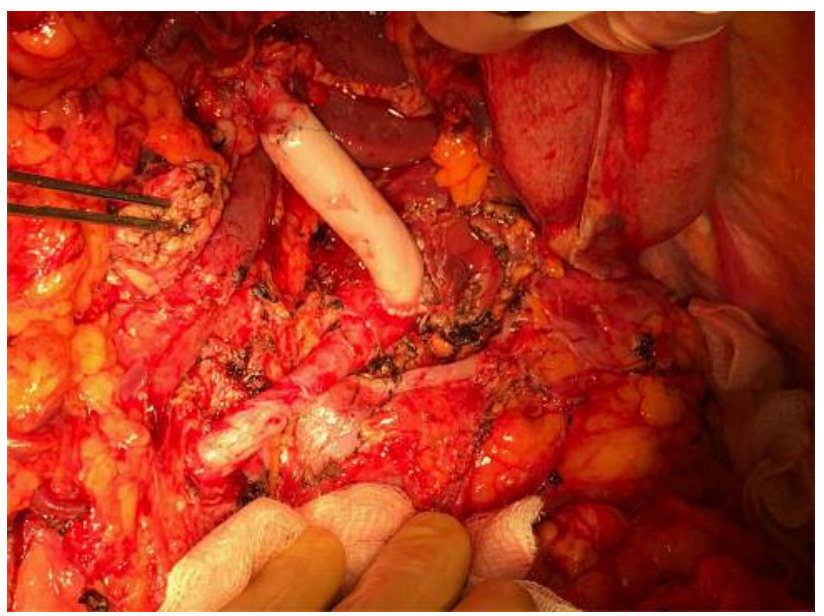

Figure 2. The final aspect after hepatic artery reconstruction using a cadaveric graft. The grasper is pointing out the pancreatic stump. term outcome, the necessity of portal vein resection not being associated with a scarcer outcome. As for the necessity of arterial reconstruction, the authors reported that none of the above-mentioned cases required such a reconstruction due to the association of a patent system of collateral circulation. Moreover, in cases in which collateral circulation did not prove to be sufficient at the preoperative investigations the authors chose to perform a preoperative coil embolization of the common hepatic artery in order to increase the collateral blood flow through the pancreaticoduodenal arcades from the superior mesenteric artery (22).

In order to diminish the risk of postoperative complications in cases in which collateral circulation does not seem to be sufficient, various techniques of vascular reconstruction have been performed with promising results; therefore, reconstruction of the branches of the celiac axis has been proposed by placing autologous grafts or allografts.

Another extremely interesting method of arterial reconstruction in cases in which the collateral flow is adequate after celiac axis resection has been reported by Machado et al. and consists of a left external iliac - hepatic arterial bypass using a Dacron prosthesis; the method proved to be successful after a modified Appleby procedure for locally advanced pancreatic cancer, the patient being asymptomatic at 13 months follow-up (23). However, placing synthetic prosthesis is still associated in certain situations with supplementary postoperative thrombotic and infectious risks; therefore attention was focused on imaging other reconstructive procedures which are able to use autologous grafts.

One of the first studies which demonstrated the utility of autologous grafts in cases in which a weak flow was identified at the level of the hepatic artery after celiac axis resection was conducted by Konisi et al.; in that study the authors reported successful arterial reconstruction using the splenic artery from the resected specimen (24).

In a more recent study conducted by Sperti et al. arterial reconstruction of the common hepatic artery after celiac axis resection was required in one out of the five cases they reported; in the meantime, the same author performed a literature review and underlined the fact that 13 out of the 90 reviewed cases necessitated association of vascular reconstructions. According to that study, reconstruction consisted most often in direct reimplantation of the common hepatic artery or by placing an arterial or a venous graft (25).

An extremely interesting study which aimed to identify which cases might necessitate common hepatic artery reconstruction after celiac axis resection for locally advanced pancreatic cancer has been published by Mittal et al. in 2015; in that paper the authors included seven patients submitted to distal pancreatectomy en bloc with splenectomy and celiac axis resection for locally invasive pancreatic body cancer (26). In order to identify which cases should be submitted to common hepatic artery reconstruction, the authors measured the pressure at the level of the common hepatic artery before and after clamping the celiac axis; therefore they observed that cases in which the mean arterial pressure at the level of the common hepatic artery drooped with more than $25 \%$ after clamping, when compared to the pre-clamping value, arterial reconstruction should be performed. Among the seven cases included in that study the authors reported this phenomenon in two cases which were further submitted to arterial reconstruction; in the first case a saphenous vein graft was used, while in the second case a polytetrafluoroethylene prosthesis was used; similarly to our case, one of the included 
patients also necessitated association of portal vein resection followed by reconstruction. The authors reported favorable outcomes as well as postoperative normalization of liver tests in all cases, demonstrating the efficacy and validity of this method of identification of patients at risk to develop further liver dysfunction in the absence of arterial reconstruction (26).

\section{Conclusion}

Although a demanding procedure, celiac axis resection followed in certain cases by arterial reconstruction seems to be safe and effective in order to achieve a radical surgical procedure for locally advanced pancreatic body cancer. Moreover association of portal vein resection seems not to influence the early or the long term outcomes.

\section{Conflicts of Interest}

The Authors have no conflicts of interest to declare regarding this study.

\section{Authors' Contributions}

VB, IB, FI - performed the surgical procedure; IB, reviewed literature data and prepared the draft of the manuscript; NB reviewed the final version of the manuscript. All Authors read and approved the final version of the manuscript.

\section{Acknowledgements}

This work was supported by the project entitled "Multidisciplinary Consortium for Supporting the Research Skills in Diagnosing, Treating and Identifying Predictive Factors of Malignant Gynecologic Disorders", project number PN-III-P1-1.2-PCCDI2017-0833.

\section{References}

1 Jemal A, Siegel R, Xu J and Ward E: Cancer statistics, 2010. CA Cancer J Clin 60(5): 277-300, 2010. PMID: 20610543. DOI: $10.3322 /$ caac. 20073

2 Sohn TA, Yeo CJ, Cameron JL, Koniaris L, Kaushal S, Abrams RA, Sauter PK, Coleman J, Hruban RH and Lillemoe KD: Resected adenocarcinoma of the pancreas-616 patients: results, outcomes, and prognostic indicators. J Gastrointest Surg 4(6): 567-579, 2000. PMID: 11307091. DOI: 10.1016/s1091$255 \mathrm{x}(00) 80105-5$

3 Fabre JM, Houry S, Manderscheid JC, Huguier M and Baumel H: Surgery for left-sided pancreatic cancer. Br J Surg 83(8): 10651070, 1996. PMID: 8869304. DOI: 10.1002/bjs.1800830810

4 NCCN Clinical Practice Guidelines in Oncology Pancreatic Adenocarcinoma, version 2. 2016. Available at: https://www.trikobe.org/ncen/guideline/

5 Okada K, Kawai M, Tani M, Hirono S, Miyazawa M, Shimizu A, Kitahata Y and Yamaue H: Surgical strategy for patients with pancreatic body/tail carcinoma: who should undergo distal pancreatectomy with en-bloc celiac axis resection? Surgery 153(3): 365-372, 2013. PMID: 23046987. DOI: 10.1016/j.surg.2012.07.036
6 Nakamura $\mathrm{T}$, Hirano $\mathrm{S}$, Noji $\mathrm{T}$, Asano $\mathrm{T}$, Okamura $\mathrm{K}$, Tsuchikawa T, Murakami S, Kurashima Y, Ebihara Y, Nakanishi Y, Tanaka K and Shichinohe T: Distal pancreatectomy with en bloc celiac axis resection (modified Appleby procedure) for locally advanced pancreatic body cancer: a single-center review of 80 consecutive patients. Ann Surg Oncol 23(Suppl 5): 969975, 2016. PMID: 27495282. DOI: 10.1245/s10434-016-5493-8

7 Ninomiya M, Tomino T, Matono R, Motomura T, Uchiyama H and Nishizaki T: Clip on staple method reduces clinically relevant pancreatic fistula after distal pancreatectomy. Anticancer Res 39(12): 6799-6806, 2019. PMID: 31810945. DOI: 10.21873/anticanres.13895

8 Kawaida H, Kono H, Amemiya H, Hosomura N, Saito R, Takahashi K, Yamamoto A, Watanabe M, Furuya S, Shimizu H, Akaike H, Kawaguchi Y, Sudo M, Matusda M, Itakura J, Fujii and Ichikawa D: Use of a reinforced triple-row stapler following distal pancreatectomy reduces the incidence of postoperative pancreatic fistula in patients with a high BMI. Anticancer Res 39(2): 1013-1018, 2019. PMID: 30711989. DOI: 10.21873/ anticanres.13207

9 Bacalbasa N, Balescu I, Vilcu M, Croitoru A, Dima S, Brasoveanu V, Brezean I and Popescu I: Pancreatoduodenectomy after neoadjuvant chemotherapy for locally advanced pancreatic cancer in the presence of an aberrant right hepatic artery. In Vivo 34(1): 401-406, 2020. PMID: 31882506. DOI: 10.21873/invivo.11788

10 Bacalbasa N, Balescu I, Dima S, Croitoru A, Brasoveanu V, Vilcu $\mathrm{M}$ and Brezean I: Pancreatoduodenectomy en bloc with vascular resections in borderline resectable pancreatic cancer. In Vivo 33(6): 2303-2308, 2019. PMID: 31662572. DOI: 10.21873/invivo.11738

11 Bacalbasa N, Balescu I, Tanase A, Brezean I, Vilcu M and Brasoveanu V: Successful resection of a non-functional paraganglioma with celiac trunk invasion followed by common hepatic artery reimplantation - a case report and literature review. In Vivo 32(4): 911-914, 2018. PMID: 29936479. DOI: 10.21873/invivo. 11328

12 Bacalbasa N, Balescu I, Tanase A, Pautov M, Brezean I, Vilcu $\mathrm{M}$ and Brasoveanu V: Spleno-pancreatectomy en bloc with parcelar gastrectomy for splenic artery aneurysm - a case report and literature review. In Vivo 32(4): 915-919, 2018. PMID: 29936480. DOI: 10.21873 /invivo. 11329

13 Bacalbasa N, Balescu I and Brasoveanu V: Celiac trunk stenosis treated by resection and splenic patch reconstruction - a case report and literature review. In Vivo 32(3): 699-702, 2018. PMID: 29695581. DOI: 10.21873/invivo.11296

14 Bacalbasa N, Brezean I, Anghel C, Barbu I, Pautov M, Balescu I and Brasoveanu V: Successful resection and vascular ligation of a large hepatic artery aneurysm - a case report and literature review. In Vivo 31(1): 979-982, 2017. PMID: 28882969. DOI: 10.21873/invivo.11157

15 Bacalbasa N, Brezean I, Anghel C, Barbu I, Pautov M, Balescu $\mathrm{I}$ and Brasoveanu V: Management of a fulminant upper gastrointestinal bleeding exteriorized through hemobilia due to arteriobiliary fistula between the common bile duct and a right hepatic artery aneurysm - a case report. In Vivo 31(5): 983-989, 2017. PMID: 28882970. DOI: 10.21873 /invivo. 11158

16 Marsoner K, Langeder R, Csengeri D, Sodeck G, Mischinger HJ and Kornprat P: Portal vein resection in advanced pancreatic adenocarcinoma: is it worth the risk? Wien Klin Wochenschr 128(15-16): 566-572, 2016. PMID: 27363995. DOI: 10.1007/ s00508-016-1024-7 
17 Fang JZ, Lu CD, Wu SD, Huang J and Zhou J: Portal vein/superior mesenteric vein resection in pancreatic cancer treatment in the elderly. Medicine (Baltimore) 96(27): e7335, 2017. PMID: 28682880. DOI: 10.1097/MD.0000000000007335

18 Nigri G, Petrucciani N, Pinna AD, Ravaioli M, Jovine E, Minni F, Grazi GL, Chirletti P, Balzano G, Ferla F, De Carlis L, Tisone G, Napoli N, Boggi U and Ramacciato G: Evolution of pancreatectomy with en bloc venous resection for pancreatic cancer in Italy. Retrospective cohort study on 425 cases in 10 pancreatic referral units. Int J Surg 55: 103-109, 2018. PMID: 29803770. DOI: 10.1016/j.ijsu.2018.05.025

19 Tee MC, Krajewski AC, Groeschl RT, Farnell MB, Nagorney DM, Kendrick ML, Cleary SP, Smoot RL, Croome KP and Truty MJ: Indications and perioperative outcomes for pancreatectomy with arterial resection. J Am Coll Surg 227(2): 255-269, 2018. PMID: 29752997. DOI: 10.1016/j.jamcollsurg.2018.05.001

20 Appleby LH: The coeliac axis in the expansion of the operation for gastric carcinoma. Cancer 6(4): 704-707, 1953. PMID: 13059764. DOI: 10.1002/1097-0142(195307)6:4<704::aidcncr2820060410>3.0.co;2-p

21 Gagandeep S, Artinyan A, Jabbour N, Mateo R, Matsuoka L, Sher L, Genyk Y and Selby R: Extended pancreatectomy with resection of the celiac axis: the modified Appleby operation. Am J Surg 192(3): 330-335, 2006. PMID: 16920427. DOI: 10.1016/j.amjsurg.2006.05.010

22 Miura T, Hirano S, Nakamura T, Tanaka E, Shichinohe T, Tsuchikawa T, Kato K, Matsumoto J and Kondo S: A new preoperative prognostic scoring system to predict prognosis in patients with locally advanced pancreatic body cancer who undergo distal pancreatectomy with en bloc celiac axis resection: a retrospective cohort study. Surgery 155(3): 457-467, 2014. PMID: 24462074. DOI: 10.1016/j.surg.2013.10.024
23 Machado MA, Surjan RC, Nishinari K, Makdissi FF and Machado MC: Iliac-hepatic arterial bypass for compromised collateral flow during modified Appleby operation for advanced pancreatic cancer. Eur J Surg Oncol 35(10): 1124-1127, 2009. PMID: 19410414. DOI: 10.1016/j.ejso.2009.04.005

24 Konishi M, Kinoshita T, Nakagori T, Inoue K, Oda T, Kimata T, Kikuchi $\mathrm{H}$ and Ryu M: Distal pancreatectomy with resection of the celiac axis and reconstruction of the hepatic artery for carcinoma of the body and tail of the pancreas. J Hepatobiliary Pancreat Surg 7(2): 183-187, 2000. PMID: 10982611. DOI: $10.1007 / \mathrm{s} 005340050173$

25 Sperti C, Berselli M and Pedrazzoli S: Distal pancreatectomy for body-tail pancreatic cancer: is there a role for celiac axis resection? Pancreatology 10(4): 491-498, 2010. PMID: 20720451. DOI: $10.1159 / 000276984$

26 Mittal A, de Reuver PR, Shanbhag S, Staerkle RF, Neale M, Thoo C, Hugh TJ, Gill AJ and Samra JS: Distal pancreatectomy, splenectomy, and celiac axis resection (DPS-CAR): common hepatic arterial stump pressure should determine the need for arterial reconstruction. Surgery 157(4): 811-817, 2015. PMID: 25532436. DOI: $10.1016 /$ j.surg.2014.10.006

Received February 28, 2020

Revised March 21, 2020

Accepted March 26, 2020 